Statistics in the Twenty-First Century: Special Volume

In Honour of Distinguished Professor Dr. Mir Masoom Ali

On the Occasion of his 75th Birthday Anniversary

PJSOR, Vol. 8, No. 3, pages 583-592, July 2012

\title{
An Inventory Model for Deteriorating Items with Permissible Delay in Payment and Inflation Under Price Dependent Demand
}

\author{
Manisha Pal \\ Department of Statistics \\ University of Calcutta, India \\ manishapal2@gmail.com \\ Hare Krishna Maity \\ Department of Statistics \\ University of Calcutta, India \\ hkmaity@gmail.com
}

\begin{abstract}
The paper studies an inventory model for deteriorating items when demand for the item is dependent on the selling price. Shortages are allowed and backlogged, and price inflation is taken into consideration. Further, it is assumed that the supplier permits the inventory manager to settle his accounts within a given specified time period. Numerical examples are cited to illustrate the model and to study the sensitivity of the model to change in model parameters.
\end{abstract}

Keywords and phrases: Inventory; Delay in payment; Deteriorating items; Backlogging of shortage; Selling price dependent demand; Inflation.

\section{Introduction}

In classical inventory models it is generally assumed that the inventory manager settles his account with the supplier as soon as the ordered quantity arrives. However, in today's business transactions it is frequently observed that the supplier allows his customer a grace period within which he can repay his dues without having to pay any interest, or may delay the payment beyond the permitted time in which case interest is charged. Since, before settling the account with the supplier, the inventory manager can sell the goods, accumulate revenue and earn interest, it makes economic sense for the manager to delay the settlement of his account to the last day of the permissible settlement period. Goyal (1985) first developed an EOQ model under the condition of permissible delay in payments. Shinn et al. (1996) extended the model by considering quantity discount for freight cost. Aggarwal and Jaggi (1995) and Hwang and Shinn (1997) extended Goyal's model to consider deterministic inventory model with constant rate of deterioration. Later Jamal et al. (1997) extended Aggarwal and Jaggi's model to allow for shortages. Pal and Ghosh $(2006,2007)$ studied deterministic inventory models with quantity dependent permissible delay period. Shah and Shah (1998) developed probabilistic inventory model for deteriorating items when delay in payment is permitted. Ghosh (2008) investigated a stochastic inventory model with stock dependent demand under conditions of permissible delay in payments. 
The above models were developed under the assumption that inflation does not play a significant role on the inventory policy. However, from financial point of view, one may consider an inventory to be a capital investment, and, as such, it should compete with other assets for an organization's limited capital fund. It is, therefore, important to investigate how time-value of money influences various inventory policies. The first study in this direction has been reported by Buzacott (1975), who considered EOQ model with inflation, subject to different types of pricing policies. Misra (1979) developed a discounted-cost model and included internal (company) and external (general economy) inflation rates for various costs associated with an inventory system. Sarker and Pan (1994) surveyed the effects of inflation and the time value of money on order quantity with finite replenishment rate. Some studies were also conducted with variable demand, see, for example, Uthayakumar and Geetha (2009), Maity (2010), Vrat and Padmanabhan (1990), Datta and Pal (1991), Hariga (1995), Hariga and Ben-Daya (1996) and Chung (2003).

In this paper, we consider a dynamic inventory model for deteriorating items allowing shortages and under inflation, when demand is price dependent and the inventory manager enjoys a fixed permissible delay in payment. The paper is organized as follows. In section 2, we analyze the model. In section 3, examples are cited and a sensitivity analysis of the model is carried out. Finally, in section 4, a discussion on the model is given.

\section{The Mathematical Model and Its Analysis}

The following notations have been used in the study:

$H \quad$ : The finite planning horizon

$r \quad$ : The constant inflation rate, $0<r<1$

$p(t) \quad$ : The selling price at time $t, p(0)=p$

$R(t) \quad$ : The price dependent demand rate at time $t$

$\theta \quad:$ The constant deterioration rate

$M \quad$ : The permissible delay in payment

$I_{r} \quad$ : The interest charged per unit of money per annum by the supplier

$I_{e} \quad:$ The interest earned per unit of money per annum

$T \quad$ : Length of a replenishment cycle

$T_{1} \quad$ : Time to exhaust stock within a replenishment cycle, $0 \leq T_{1}<T$

$A \quad$ : The ordering cost per order at time $t=0$

$c \quad$ : Purchase cost per unit at time $t=0$

$I \quad$ : Fraction of the purchase cost per unit defining the holding cost per unit per annum

$s \quad$ : shortage cost per unit per annum at time $t=0$ 
The demand rate at time $t$ is given by

$$
\begin{aligned}
R(t) & =a-b p e^{r t}, \quad t \leq \frac{1}{r} \log \left(\frac{a}{b p}\right) \\
& =0, \text { for } t>\frac{1}{r} \log \left(\frac{a}{b p}\right),
\end{aligned}
$$

where $a, b \geq 0$.

We take the length $H$ of the planning horizon to be such that the demand rate at the end of the planning horizon remains non-negative, that is, $H \leq \frac{1}{r} \log \left(\frac{a}{b p}\right)$. This may also be ensured by taking $a \succ \succ b$.

We assume that the planning horizon is divided into $n$ reorder intervals of length $T$, so that we have $H=n T$. Further, the costs and selling price during a reorder cycle is assumed to remain the same as that at the beginning of the cycle. Thus, the price in the $s^{\text {th }}$ cycle is given by $p e^{r(s-1) T}$, and hence the demand rate is

$$
R(t)=a-b p e^{r(s-1) T},(s-1) T \leq t<s T, 1 \leq s \leq n .
$$

The inventory policy is to place an order at the beginning of each reorder interval, and the order quantity is just sufficient to meet the backorders in the previous period and the demand during the first $T_{1}$ units of time in the current period.

Let, $I_{s}(t)$ denote the inventory level at time point $t$ in the $s^{\text {th }}$ cycle, $1 \leq s \leq n$. Since depletion of stock occurs owing to demand and deterioration, the following differential equations define transitions in inventory:

$$
\begin{aligned}
& \frac{d I_{s}(t)}{d t}+\theta I_{s}(t)=-a+b p e^{r(s-1) T}, 0 \leq t \leq T_{1} 1 \leq s \leq n \\
& \frac{d I_{s-1}(t)}{d t}=-a+b p e^{r(s-1) T}, T_{1} \leq t \leq T 1 \leq s \leq n
\end{aligned}
$$

where $I_{s}\left(T_{1}\right)=0$, for $1 \leq s \leq n$.

Solving the differential equations, we get

$$
I_{s}(t)=\left\{\begin{array}{cc}
\frac{D_{s}}{\theta}\left(1-e^{\theta\left(T_{1}-t\right)}\right), & 0 \leq t \leq T_{1}, 1 \leq s \leq n \\
D_{s}\left(t-T_{1}\right), & T_{1} \leq t \leq T, 1 \leq s \leq n,
\end{array}\right.
$$

where $D_{s}=-a+b p e^{r(s-1) T}$.

The different costs incurred during the planning horizon are as follows:

(i) Total ordering cost:

$$
\begin{aligned}
& A_{H}\left(T, T_{1}\right)=A+A e^{r T}+\cdots+A e^{r(n-1) T} \\
= & A \frac{e^{r H}-1}{e^{r T}-1}
\end{aligned}
$$


(ii) Total purchasing cost:

$$
\begin{aligned}
& C_{H}\left(T, T_{1}\right)=c I_{0}(0)+c e^{r T}\left(I_{1}(0)+\int_{T_{1}}^{T}(a-b p) d t\right)+\cdots+c e^{r(n-1) T}\left(I_{n-2}(0)+\int_{T_{1}}^{T}\left(a-b p e^{r(n-1) T}\right) d t\right) \\
& =\frac{c}{\theta}\left(e^{\theta T_{1}}-1\right)\left(a \frac{e^{r H}-1}{e^{r T}-1}-b p \frac{e^{2 r H}-1}{e^{2 r T}-1}\right)+c\left(T-T_{1}\right)\left(a \frac{e^{r H-r T}-1}{e^{r T}-1}-b p \frac{e^{2 r H-2 r T}-1}{e^{2 r T}-1}\right)
\end{aligned}
$$

(iii) Total holding cost:

$$
\begin{gathered}
K_{H}\left(T, T_{1}\right)=I c \int_{0}^{T_{1}} I_{0}(t) d t+I c e^{r T} \int_{0}^{T_{1}} I_{t}(t) d t+\cdots+I c e^{r(n-1) T} \int_{0}^{T_{1}} I_{n-1}(t) d t \\
=\frac{I c}{\theta^{2}}\left(e^{\theta T_{1}}-\theta T_{1}-1\right)\left(a \frac{e^{r H}-1}{e^{r T}-1}-b p \frac{e^{2 r H}-1}{e^{2 r T}-1}\right)
\end{gathered}
$$

(iv) Total deterioration cost:

$$
\begin{aligned}
& D_{H}\left(T, T_{1}\right)=\theta c \int_{0}^{T_{1}} I_{0}(t) d t+\theta c e^{r T} \int_{0}^{T_{1}} I_{t}(t) d t+\cdots+\theta c e^{r(n-1) T} \int_{0}^{T_{1}} I_{n-1}(t) d t \\
& =\frac{c}{\theta}\left(e^{\theta T_{1}}-\theta T_{1}-1\right)\left(a \frac{e^{r H}-1}{e^{r T}-1}-b p \frac{e^{2 r H}-1}{e^{2 r T}-1}\right)
\end{aligned}
$$

(v) Total shortage cost:

$$
\begin{aligned}
& S_{H}\left(T, T_{1}\right)=-s\left(I_{0}(T)+e^{r T} I_{1}(T)+\cdots+e^{r(n-1) T} I_{n-1}(T)\right) \\
& =s\left(T-T_{1}\right)\left(a \frac{e^{r H}-1}{e^{r T}-1}-b p \frac{e^{2 r H}-1}{e^{2 r T}-1}\right)
\end{aligned}
$$

(v) Total selling price:

$$
\begin{aligned}
& P_{H}\left(T, T_{1}\right)=p \int_{0}^{T_{1}}(a-b p) d t+p e^{r T}\left(\int_{0}^{T_{1}}\left(a-b p e^{r T}\right) d t-I_{0}(T)\right) \\
& +\cdots+p e^{r(n-1) T}\left(\int_{0}^{T_{1}}\left(a-b p e^{r(n-1) T}\right) d t-I_{n-2}(T)\right) \\
& =p T_{1}\left(a \frac{e^{r H}-1}{e^{r T}-1}-b p \frac{e^{2 r H}-1}{e^{2 r T}-1}\right)-p e^{r T}\left(T-T_{1}\right)\left(a \frac{e^{r H-r T}-1}{e^{r T}-1}-b p \frac{e^{2 r H-2 r T}-1}{e^{2 r T}-1}\right)
\end{aligned}
$$

(vi) Total interest earned:

Since the inventory manager can earn revenue by selling his goods when $M \leq T_{1}$, we have $I E_{H}\left(T, T_{1}\right)=$ total interest earned during $(0, H)$

$=p I_{e} \int_{0}^{T_{1}}(a-b p)\left(T_{1}-t\right) d t+p I_{e} e^{r T} \int_{0}^{T_{1}}\left(a-b p e^{r T}\right)\left(T_{1}-t\right) d t$ 


$$
\begin{aligned}
& +\ldots+p I_{e} e^{r(n-1) T} \int_{0}^{T_{1}}\left(a-b p e^{r(n-1) T}\right)\left(T_{1}-t\right) d t \text {, when } M \leq T_{1} \\
& \text { =0, } \quad \text { when } M \geq T_{1} \\
& =p I_{e} \frac{T_{1}^{2}}{2}\left(a \frac{e^{r H}-1}{e^{r T}-1}-b p \frac{e^{2 r H}-1}{e^{2 r T}-1}\right) \text {, when } M \leq T_{1} \\
& =0, \quad \text { when } M \geq T_{1}
\end{aligned}
$$

(vii) Total interest payable:

$$
\begin{gathered}
I P_{H}\left(T, T_{1}\right)=c I_{r} \int_{M}^{T_{1}} I_{0}(t) d t+c I_{r} e^{r T} \int_{M}^{T_{1}} I_{1}(t) d t+\cdots+c I_{r} e^{(n-1) r T} \int_{M}^{T_{1}} I_{n-1}(t) d t \\
=\frac{c I_{r}}{\theta}\left(e^{\theta\left(T_{1}-M\right)}-\theta\left(T_{1}-M\right)-1\right)\left(a \frac{e^{r H}-1}{e^{r T}-1}-b p \frac{e^{2 r H}-1}{e^{2 r T}-1}\right), \text { when } M \leq T_{1} \\
=c I_{r}\left(\int_{0}^{T_{1}}(a-b p)\left(T_{1}-t\right) d t+\int_{T_{1}}^{M}(a-b p)(M-t) d t\right) \\
+c I_{r} e^{r T}\left(\int_{0}^{T_{1}}\left(a-b p e^{r T}\right)\left(T_{1}-t\right) d t+\int_{T_{1}}^{M}\left(a-b p e^{r T}\right)(M-t) d t\right) \\
+\ldots+c I_{r} e^{r(n-1) T}\left(\int_{0}^{T_{1}}\left(a-b p e^{r(n-1) T}\right)\left(T_{1}-t\right) d t+\int_{T_{1}}^{M}\left(a-b p e^{r(n-1) T}\right)(M-t) d t\right) \\
=c I_{r}\left(a \frac{e^{r H}-1}{e^{r T}-1}-b p \frac{e^{2 r H}-1}{e^{2 r T}-1}\right)\left(\frac{T_{1}^{2}}{2}+\frac{\left(M-T_{1}\right)^{2}}{2}\right), \text { when } M \geq T_{1} .
\end{gathered}
$$

Hence, the total profit made in the interval $[0, H]$ is:

$$
\begin{aligned}
C^{M}\left(T, T_{1}\right) & =C_{1}^{M}\left(T, T_{1}\right), \text { for } M \leq T_{1} \\
& =C_{2}^{M}\left(T, T_{1}\right), \text { for } M \geq T_{1},
\end{aligned}
$$

where

$$
\begin{aligned}
& C_{1}^{M}\left(T, T_{1}\right)=\left[p T_{1}+p I_{e} \frac{T_{1}^{2}}{2}-\left(e^{\theta T_{1}}-\theta T_{1}-1\right)\left(\frac{I c}{\theta^{2}}+\frac{c}{\theta}\right)-\frac{c I_{r}}{\theta}\left(e^{\theta\left(T_{1}-M\right)}-\theta\left(T_{1}-M\right)-1\right)\right. \\
& \left.-s\left(T-T_{1}\right)-\frac{c}{\theta}\left(e^{\theta T_{1}}-1\right)\right]\left(a \frac{e^{r H}-1}{e^{r T}-1}-b p \frac{e^{2 r H}-1}{e^{2 r T}-1}\right) \\
& -\left(T-T_{1}\right)\left(c+p e^{r T}\right)\left(a \frac{e^{r H-r T}-1}{e^{r T}-1}-b p \frac{e^{2 r H-2 r T}-1}{e^{2 r T}-1}\right)-A \frac{e^{r H}-1}{e^{r T}-1} \\
& C_{2}^{M}\left(T, T_{1}\right)=\left[p T_{1}-c I_{r}\left(\frac{T_{1}^{2}}{2}+\frac{\left(M-T_{1}\right)^{2}}{2}\right)-\left(e^{\theta T_{1}}-\theta T_{1}-1\right)\left(\frac{I c}{\theta^{2}}+\frac{c}{\theta}\right)\right.
\end{aligned}
$$




$$
\begin{aligned}
& -\frac{c I_{r}}{\theta}\left(e^{\theta\left(T_{1}-M\right)}-\theta\left(T_{1}-M\right)-1\right)-s\left(T-T_{1}\right)-\frac{c}{\theta}\left(e^{\theta T_{1}}-1\right)\left(a \frac{e^{r H}-1}{e^{r T}-1}-b p \frac{e^{2 r H}-1}{e^{2 r T}-1}\right) \\
& -\left(T-T_{1}\right)\left(c+p e^{r T}\right)\left(a \frac{e^{r H-r T}-1}{e^{r T}-1}-b p \frac{e^{2 r H-2 r T}-1}{e^{2 r T}-1}\right)-A \frac{e^{r H}-1}{e^{r T}-1}
\end{aligned}
$$

Lemma 1: Both $C_{1}^{M}\left(T, T_{1}\right)$ and $C_{2}^{M}\left(T, T_{1}\right)$ are decreasing function of $I$ and $s$, for fixed $T_{1}$ and $T$.

Proof: We have,

$$
\begin{aligned}
& \frac{\partial C_{1}^{M}\left(T_{1}, T\right)}{\partial I}=-\frac{c}{\theta^{2}}\left(e^{\theta T_{1}}-\theta T_{1}-1\right)\left(a \frac{e^{r H}-1}{e^{r T}-1}-b p \frac{e^{2 r H}-1}{e^{2 r T}-1}\right) \leq 0 \\
& \frac{\partial C_{2}^{M}\left(T_{1}, T\right)}{\partial I}=-\frac{c}{\theta^{2}}\left(e^{\theta T_{1}}-\theta T_{1}-1\right)\left(a \frac{e^{r H}-1}{e^{r T}-1}-b p \frac{e^{2 r H}-1}{e^{2 r T}-1}\right) \leq 0
\end{aligned}
$$

and,

$$
\begin{aligned}
& \frac{\partial C_{1}^{M}\left(T_{1}, T\right)}{\partial s}=-\left(T-T_{1}\right)\left(a \frac{e^{r H}-1}{e^{r T}-1}-b p \frac{e^{2 r H}-1}{e^{2 r T}-1}\right) \leq 0 \\
& \frac{\partial C_{2}^{M}\left(T_{1}, T\right)}{\partial s}=-\left(T-T_{1}\right)\left(a \frac{e^{r H}-1}{e^{r T}-1}-b p \frac{e^{2 r H}-1}{e^{2 r T}-1}\right) \leq 0
\end{aligned}
$$

Thus, the total profit decreases with increase in the inventory holding cost and the shortage cost.

Lemma2: $C_{1}^{M}\left(T, T_{1}\right)$ and $C_{2}^{M}\left(T, T_{1}\right)$ are both concave in $T_{1}$, for given $T$.

Proof: We have

$$
\begin{aligned}
& \frac{\partial^{2} C_{1}^{M}\left(T_{1}, T\right)}{\partial T_{1}^{2}}=-\left(a \frac{e^{r H}-1}{e^{r T}-1}-b p \frac{e^{2 r H}-1}{e^{2 r T}-1}\right) \frac{T^{2}}{H}\left(c e^{\theta T}(I+2)+c I_{r} e^{\theta\left(T_{1}-M\right)}-p I_{e}\right) \leq 0, \text { since } \\
& \left(a \frac{e^{r H}-1}{e^{r T}-1}-b p \frac{e^{2 r H}-1}{e^{2 r T}-1}\right) \geq 0 \text { and }\left(c e^{\theta T}(I+2)+c I_{r} e^{\theta\left(T_{1}-M\right)}-p I_{e}\right) \geq 0 .
\end{aligned}
$$

Hence $C_{1}^{M}\left(T_{1}, T\right)$ is a concave function of $T_{1}$, for given $T$.

Similarly it can be shown that $C_{2}^{M}\left(T_{1}, T\right)$ is concave in $T_{1}$, for given $T$.

The optimal values of $\left(T_{1}, T\right)$ are obtained so as to maximize $C^{M}\left(T_{1}, T\right)$. 
It may be noted that the optimal values of $\left(T_{1}, T\right)$ maximizing $C_{1}^{M}\left(T_{1}, T\right)$ satisfy $\frac{\partial C_{1}^{M}\left(T, T_{1}\right)}{\partial T_{1}}=0$ and $\frac{\partial C_{1}^{M}\left(T, T_{1}\right)}{\partial T}=0$, which reduce to the following equations:

$$
\begin{aligned}
& c\left(2+I_{r} e^{-\theta M}+\frac{I}{\theta}\right) e^{\theta T_{1}}-p I_{e} T_{1}-\left(c I_{r}+p+s\right)=\frac{\left(c+p e^{r T}\right)\left(a \frac{e^{r H-r T}-1}{e^{r T}-1}-b p \frac{e^{2 r H-2 r T}-1}{e^{2 r T}-1}\right)}{\left(a \frac{e^{r H}-1}{e^{r T}-1}-b p \frac{e^{2 r H}-1}{e^{2 r T}-1}\right)} \\
& \left(T-T_{1}\right)\left(c+p e^{r T}\right)\left(a \frac{r e^{r H-r T}\left(e^{r T}-1\right)+\left(e^{r H-r T}-1\right) r e^{r T}}{\left(e^{r T}-1\right)^{2}}-2 r b p \frac{e^{r H-r T}\left(e^{2 r T}-1\right)+\left(e^{2 r H-2 r T}-1\right) e^{2 r T}}{\left(e^{2 r T}-1\right)^{2}}\right)- \\
& {\left[p T_{1}+p I_{e} \frac{T_{1}^{2}}{2}-\left(e^{\theta T_{1}}-\theta T_{1}-1\right)\left(\frac{I c}{\theta^{2}}+\frac{c}{\theta}\right)-\frac{c I_{r}}{\theta}\left(e^{\theta\left(T_{1}-M\right)}-\theta\left(T_{1}-M\right)-1\right)-s\left(T-T_{1}\right)\right.} \\
& \left.-\frac{c}{\theta}\left(e^{\theta T_{1}}-1\right)\right]\left(a \frac{e^{r H}-1}{\left(e^{r T}-1\right)^{2}} r e^{r T}-b p \frac{e^{2 r H}-1}{\left(e^{2 r T}-1\right)^{2}} 2 r e^{2 r T}\right)-\left(c+p e^{r T}\right) \times \\
& \left(a \frac{e^{r H-r T}-1}{e^{r T}-1}-b p \frac{e^{2 r H-2 r T}-1}{e^{2 r T}-1}\right)-p r e^{r T}\left(T-T_{1}\right)\left(a \frac{e^{r H-r T}-1}{e^{r T}-1}-b p \frac{e^{2 r H-2 r T}-1}{e^{2 r T}-1}\right) \\
& \left.-A \frac{e^{r H}-1}{\left(e^{r T}-1\right)^{2}} r e^{r T}\right]\left(a \frac{e^{r H}-1}{e^{r T}-1}-b p \frac{e^{2 r H}-1}{e^{2 r T}-1}\right)^{-1}=s .
\end{aligned}
$$

Similarly, the optimal values of $\left(T_{1}, T\right)$ maximizing $C_{2}^{M}\left(T_{1}, T\right)$ satisfy $\frac{\partial C_{1}^{M}\left(T, T_{1}\right)}{\partial T_{1}}=0$ and $\frac{\partial C_{1}^{M}\left(T, T_{1}\right)}{\partial T}=0$, which reduce to the following equations:

$$
\begin{aligned}
& p+s-c\left[I_{r}(M-1)-1-\frac{I}{\theta}\right]+c T_{1}(I+\theta)-c e^{\theta T_{1}}\left(2+\frac{I}{\theta}+I_{r} e^{-\theta M}\right) \\
& =\left(c+p e^{r T}\right) \frac{\left(a \frac{e^{r H-r T}-1}{e^{r T}-1}-b p \frac{e^{2 r H-2 r T}-1}{e^{2 r T}-1}\right)}{\left(a \frac{e^{r H}-1}{e^{r T}-1}-b p \frac{e^{2 r H}-1}{e^{2 r T}-1}\right)}
\end{aligned}
$$




$$
\begin{aligned}
& \left(c I_{r}\left(\frac{T_{1}^{2}}{2}+\frac{\left(M-T_{1}\right)^{2}}{2}\right)+\left(e^{\theta T_{1}}-\theta T_{1}-1\right)\left(\frac{I c}{\theta^{2}}+\frac{c}{\theta}\right)-p T_{1}+\frac{c I_{r}}{\theta}\left(e^{\theta\left(T_{1}-M\right)}-\theta\left(T_{1}-M\right)-1\right)\right. \\
& \left.+s\left(T-T_{1}\right)+\frac{c}{\theta}\left(e^{\theta T_{1}}-1\right)\right)\left(a \frac{e^{r H}-1}{\left(e^{r T}-1\right)^{2}} r e^{r T}-b p \frac{e^{2 r H}-1}{\left(e^{2 r T}-1\right)^{2}} 2 r e^{2 r T}\right)-\left(c+p e^{r T}\right) \times \\
& \left(a \frac{e^{r H-r T}-1}{e^{r T}-1}-b p \frac{e^{2 r H-2 r T}-1}{e^{2 r T}-1}\right)-\left(T-T_{1}\right)\left\{p r e^{r T}\left(a \frac{e^{r H-r T}-1}{e^{r T}-1}-b p \frac{e^{2 r H-2 r T}-1}{e^{2 r T}-1}\right)\right. \\
& \left.+\left(c+p e^{r T}\right)\left(a \frac{-r e^{r H-r T}\left(e^{r T}-1\right)-\left(e^{r H-r T}-1\right) r e^{r T}}{\left(e^{r T}-1\right)^{2}}-b p \frac{-2 r e^{r H-r T}\left(e^{2 r T}-1\right)-\left(e^{2 r H-2 r T}-1\right) 2 r e^{2 r T}}{\left(e^{2 r T}-1\right)^{2}}\right)\right\} \\
& \left.-A \frac{e^{r H}-1}{\left(e^{r T}-1\right)^{2}} r e^{r T}\right]\left(a \frac{e^{r H}-1}{e^{r T}-1}-b p \frac{e^{2 r H}-1}{e^{2 r T}-1}\right)^{-1}=s .
\end{aligned}
$$

\section{Numerical Examples}

Example 1: Suppose $A=$ Rs. 250, $c=$ Rs. 20, $p=$ Rs. 24, $s=$ Re 0.1, $I=0.1, I_{e}=.12, I_{r}=.15$, $r=.02, \theta=.02, M=0.1$ year, $H=4$ years, $a=2000, b=0.1$.

Using the software MATLAB, we get the following output:

For $M \leq T_{1}$,

$$
T_{\text {lopt }}=0.226, \mathrm{~T}_{\mathrm{opt}}=0.433 \text { and } \max C_{1}^{M}\left(T_{1}, T\right)=28879.11
$$

For $M \geq T_{1}$,

$$
T_{\text {lopt }}=0.098, \mathrm{~T}_{\mathrm{opt}}=0.443 \text { and } \max C_{2}^{M}\left(T_{1}, T\right)=28060.3 .
$$

Hence, the optimal solution is $T_{1}=0.226, T=0.433$, and $C^{M}\left(T_{1}, T\right)=28879.11$.

Example 2: Suppose $A=250, c=$ Rs. 20, $p=$ Rs. 25, $s=\operatorname{Re} 0.1, I=0.1, I_{e}=.12, I_{r}=.15, r=$ $0.15, \theta=0.4, M=0.1$ year, $H=2$ years, $a=2000, b=0.1$.

MATLAB output is:

For $M \leq T_{1}, T_{1 \mathrm{opt}}=0.01, T_{\mathrm{opt}}=0.395$ and $\max C_{1}^{M}\left(T_{1}, T\right)=21067.82$;

For $M \geq T_{1}, T_{\text {lopt }}=0.002, T_{\mathrm{opt}}=0.393$ and $\max C_{2}^{M}\left(T_{1}, T\right)=21073.55$.

Hence, the optimal solution is $T_{1}=0.002, T=0.393$, and $C^{M}\left(T_{1}, T\right)=21073.55$.

Example 3: The following tables show the change in the optimal values of $T_{1}$ and $T$ with change in the model parameters $H, M, r, p$ and $\theta$. We take $A=$ Rs. 250, $c=$ Rs. 20, $s=\operatorname{Re}$ $0.1, I=0.1, I_{e}=0.12, I_{r}=.15, a=2000, b=0.1$. 
Table1: $\quad p=$ Rs.25, $r=.02, \theta=.02$.

\begin{tabular}{|c|c|c|c|c|c|c|c|c|c|c|c|c|c|c|c|}
\hline $\mathrm{H}$ & \multicolumn{3}{|c|}{$\mathrm{M}=0.01$} & \multicolumn{3}{|c|}{$\mathrm{M}=0.05$} & \multicolumn{3}{|c|}{$\mathrm{M}=0.1$} & \multicolumn{3}{|c|}{$\mathrm{M}=0.3$} & \multicolumn{3}{|c|}{$\mathrm{M}=0.6$} \\
\hline & $T_{1}$ & $\mathrm{~T}$ & Profit & $\mathrm{T}_{1}$ & $\mathrm{~T}$ & Profit & $\mathrm{T}_{1}$ & $\mathrm{~T}$ & Profit & $\mathrm{T}_{1}$ & $\mathrm{~T}$ & Profit & $\mathrm{T}_{1}$ & $\mathrm{~T}$ & Profit \\
\hline 0.8 & 0.294 & 0.294 & 8413.3 & 0.298 & 0.298 & 8630.3 & 0.311 & 0.311 & 8857.7 & 0.423 & 0.423 & 9405.8 & 0.675 & 0.675 & 9730.6 \\
\hline 1.0 & 0.294 & 0.294 & 8430.2 & 0.298 & 0.298 & 8647.6 & 0.311 & 0.311 & 8875.5 & 0.423 & 0.423 & 9424.7 & 0.675 & 0.675 & 9750.2 \\
\hline 2.0 & 0.271 & 0.299 & 8522.5 & 0.298 & 0.298 & 8735.0 & 0.311 & 0.311 & 8965.2 & 0.423 & 0.423 & 9519.9 & 0.675 & 0.675 & 9848.7 \\
\hline 4.0 & 0.165 & 0.394 & 9002.0 & 0.206 & 0.388 & 9100.9 & 0.256 & 0.379 & 9227.4 & 0.423 & 0.423 & 9714.2 & 0.675 & 0.675 & 10050 \\
\hline 6.0 & 0.124 & 0.513 & 9460.2 & 0.165 & 0.505 & 9517.2 & 0.217 & 0.495 & 9590.8 & 0.420 & 0.434 & 9914.2 & 0.675 & 0.675 & 10256 \\
\hline
\end{tabular}

Table2: $\quad p=$ Rs. $25, H=2, M=0.01$

\begin{tabular}{|c|c|c|c|c|c|c|c|c|c|c|c|c|c|c|c|}
\hline$r$ & \multicolumn{3}{|c|}{$\theta=0.02$} & \multicolumn{3}{|c|}{$\theta=0.08$} & \multicolumn{3}{|c|}{$\theta=0.15$} & \multicolumn{3}{|c|}{$\theta=0.2$} & \multicolumn{3}{|c|}{$\theta=0.4$} \\
\hline & $T_{1}$ & $\mathrm{~T}$ & Profit & $\mathrm{T}_{1}$ & $\mathrm{~T}$ & Profit & $\mathrm{T}_{1}$ & $\mathrm{~T}$ & Profit & $\mathrm{T}_{1}$ & $\mathrm{~T}$ & Profit & $\mathrm{T}_{1}$ & $\mathrm{~T}$ & Profit \\
\hline 0.02 & 0.271 & 0.299 & 8522.5 & 0.132 & 0.265 & 8210.5 & 0.083 & 0.255 & 8090 & 0.065 & 0.251 & 8046 & 0.036 & 0.246 & 7968.8 \\
\hline 0.05 & 0.217 & 0.309 & 8816.1 & 0.109 & 0.286 & 8615.9 & 0.069 & 0.278 & 8537.3 & 0.055 & 0.275 & 8508.5 & 0.030 & 0.271 & 8457.7 \\
\hline 0.08 & 0.163 & 0.327 & 9201.4 & 0.084 & 0.312 & 9091.2 & 0.054 & 0.307 & 9047.4 & 0.043 & 0.305 & 9031.3 & 0.024 & 0.302 & 9002.8 \\
\hline 0.10 & 0.125 & 0.344 & 9516.1 & 0.065 & 0.334 & 9452.7 & 0.042 & 0.330 & 9427.4 & 0.033 & 0.329 & 9418.1 & 0.018 & 0.326 & 9401.6 \\
\hline 0.12 & 0.085 & 0.364 & 9882.4 & 0.045 & 0.357 & 9854.1 & 0.029 & 0.355 & 9842.7 & 0.023 & 0.354 & 9838.6 & 0.013 & 0.353 & 9831.1 \\
\hline 0.15 & 0.019 & 0.396 & 10539 & 0.010 & 0.395 & 10538 & 0.010 & 0.395 & 10537 & 0.003 & 0.394 & 10537 & 0.002 & 0.393 & 10537 \\
\hline 0.20 & 0.010 & 0.463 & 11850 & 0.004 & 0.460 & 11866 & 0.004 & 0.46 & 11866 & 0.004 & 0.460 & 11866 & 0.004 & 0.460 & 11866 \\
\hline
\end{tabular}

Table 3: $\quad r=.02, H=4, \theta=.02$

\begin{tabular}{|c|c|c|c|c|c|c|c|c|c|c|c|c|c|c|c|}
\hline $\mathrm{M}$ & \multicolumn{3}{|c|}{$\mathrm{P}=22$} & \multicolumn{3}{|c|}{$\mathrm{P}=24$} & \multicolumn{3}{|c|}{$\mathrm{P}=26$} & \multicolumn{3}{|c|}{$\mathrm{P}=28$} & \multicolumn{3}{|c|}{$\mathrm{P}=30$} \\
\hline & $\mathrm{T}_{1}$ & $\mathrm{~T}$ & Profit & $\mathrm{T}_{1}$ & $\mathrm{~T}$ & Profit & $\mathrm{T}_{1}$ & $\mathrm{~T}$ & Profit & $\mathrm{T}_{1}$ & $\mathrm{~T}$ & Profit & $\mathrm{T}_{1}$ & $\mathrm{~T}$ & Profit \\
\hline 0.01 & 0.077 & 0.721 & 3359.0 & 0.137 & 0.446 & 7058.1 & 0.194 & 0.361 & 10985 & 0.260 & 0.326 & 15051 & 0.322 & 0.322 & 19241 \\
\hline 0.05 & 0.114 & 0.717 & 3381.5 & 0.177 & 0.441 & 7129.5 & 0.236 & 0.355 & 11114 & 0.305 & 0.319 & 15246 & 0.327 & 0.327 & 19468 \\
\hline 0.10 & 0.161 & 0.713 & 3408.8 & 0.226 & 0.433 & 7219.8 & 0.287 & 0.345 & 11280 & 0.328 & 0.328 & 15483 & 0.341 & 0.341 & 19709 \\
\hline 0.30 & 0.350 & 0.706 & 3506.0 & 0.416 & 0.416 & 7591.6 & 0.430 & 0.430 & 11837 & 0.446 & 0.446 & 16085 & 0.464 & 0.464 & 20336 \\
\hline 0.50 & 0.539 & 0.716 & 3583.4 & 0.576 & 0.576 & 7831.7 & 0.596 & 0.596 & 12111 & 0.618 & 0.618 & 16393 & 0.642 & 0.642 & 20680 \\
\hline
\end{tabular}

From the above tables we have the following observations:

(i) As $\mathrm{M}$ increases $T_{1}$ increases.

(ii) As $H$ increases $T_{1}$ decreases.

(iii) As $r$ increases $T_{1}$ decreases, but $T$ increases.

(iv) As $\theta$ increases, both $T_{1}$ and $T$ decrease.

(v) As $p$ increases, both $T_{1}$ and $T$ increase

\section{Conclusion}

The paper studies a dynamic inventory model for deteriorating items. The demand for the item is dependent on the selling price and unmet demand is backlogged. The replenishment source allows the inventory manager a certain fixed period of time to settle his accounts. No interest is charged during this period, but beyond it the manager has to pay an interest. The effect of inflation on various costs is also taken into consideration. The optimum ordering policy is determined by maximizing the total profit over the planning horizon. 


\section{References}

1. Aggarwal, S.P. and Jaggi, C.K.: Ordering policies of deteriorating items under conditions of permissible delay in payments, J. Operat. Res.Soc., 46, (1995) 658662.

2. Chung, K.J.: An algorithm for an inventory model with inventory-level-dependent demand rate, Comput. Operat. Res. (2003), 30, 1311-1317.

3. Datta, T.K. and Pal, A.K.: Effects of inflation and time-value of money on an inventory model with linear time-dependent demand rate and shortages, Eur. $J$. Operat. Res. (1991), 52: 326-333.

4. Goyal, S.K.: Economic order quantity under conditions of permissible delay in payments, J. Operat. Res.Soc., 36, (1985) 335-338.

5. Hariga, M.A.: Effects of inflation and time-value of money on an inventory model with time-dependent demand rate and shortages, Eur. J. Operat. Res. (1995), 81, 512-520.

6. Hariga, M. and Ben-Daya, M.: Optimal time-varying lot-sizing models under inflationary conditions. Eur. J. Operat. Res. (1996), 89, 313-325.

7. Hou, K.L. and Lin, L.C.: A cash flow oriented EOQ Model with deteriorating items under permissible delay in payments, Journal of Applied Science (2009), 9(9), 1791-1794.

8. Hwang, H. and Shinn, S.W.: Retailer's pricing and lot sizing policy for exponentially deteriorating product under condition of permissible delay in payments, Computer \& Operations Research, 24, (1997) 539-547.

9. Jamal, A.M., Sarker, B.R. and Wang, S.: An ordering policy for deteriorating items with allowable shortage and permissible delay in payment, J. Operat. Res.Soc., 48, (1997) 826-833.

10. Maity, A.K.: One machine multiple-product problem with production-inventory system under fuzzy inequality constraint. Applied Soft Comput. (2010), 10.1016/j.asoc. 2009. 12.029.

11. Pal, M. and Ghosh, S.K. (2006): An inventory model with shortage and quantity dependent permissible delay in payment, Aust. Soc. Operat. Res. Bull. (2006), 25(3).

12. Pal, M. and Ghosh, S.K. (2007): An inventory model for deteriorating items with quantity dependent permissible delay in payment and partial backlogging of shortage, CSA Bulletin, 59, September \& December.

13. Shah, N.H. and Shah, Y.K.: A discrete-in-time probabilistic inventory model for deteriorating items under conditions of permissible delay in payments, Inter. $J$. Syst. Sci. (1998), 29, 121-126.

14. Shinn, S.W., Hwang, H.P. and Sung, S.: Joint price and lot size determination under conditions of permissible delay in payments and quantity discounts for freight cost, Euro. J. Operat. Res., 91, (1996) 528-542.

15. Uthayakumar, R. and Geetha, K.V.: Replenishment policy for single item inventory model with money inflation, OPSEARCH (2009), 46(3), 345-357.

16. Vrat, P. and Padmanabhan, G.: An inventory model under inflation for stockdependent consumption rate items, Eng. Costs Prod. Econ. (1990), 19, 379-383. 